\title{
Intermittency and Deformation Band Propagation in an Austenitic FeMnC TWIP Steel
}

\author{
M.A. Lebyodkin ${ }^{a, *}$, T.A. LebedkinA ${ }^{a, b}$, A. Roth ${ }^{a}$ And S. Allain ${ }^{c}$ \\ ${ }^{a}$ LEM3, Université de Lorraine/CNRS UMR 7239, Ile du Saulcy, 57045 Metz, France \\ ${ }^{b}$ Institute of Solid State Physics, Russian Academy of Sciences, 142432 Chernogolovka, Russia \\ ${ }^{c}$ Arcelormittal Maizières Research SA, Voie Romaine, BP 30320, F-57283 Maizières les Metz, France
}

\begin{abstract}
Plastic deformation of austenitic FeMnC TWIP steels exhibits complex spatiotemporal patterns associated with the occurrence of deformation bands and the concomitant fluctuations of the deforming stress. Although the plastic instability is a generic property of these steels and is usually attributed to interaction of dislocations with solutes (the Portevin-Le Chatelier effect), both the kinematics of the deformation bands and the characteristic shape of stress serrations observed in the carbon-containing TWIP steel at room temperature manifest unusual features. The nature of these peculiarities and the mechanism of jerky flow in such steels are unclear. In the present work, an attempt is made to get an insight into the jerky flow in the Fe22Mn0.6C steel by studying the time series characterizing the evolution of plastic deformation at various scales: stress-time curves, local strain field, and acoustic emission accompanying the deformation processes. Stress and strain fluctuations related to two distinct scale ranges are detected and characterized.
\end{abstract}

PACS: $62.20 . \mathrm{F}-$, 81.70.- $\mathrm{q}, 05.65 .+\mathrm{b}, 62.65 .+\mathrm{k}$

\section{Introduction}

Austenitic FeMnC steels attract great attention because of their remarkable mechanical properties which are caused by action of various plasticity mechanisms (see [1] and references therein). At room temperature (RT), the stacking fault energy of Fe22Mn0.6C steel is small enough to put into competition dislocation glide and twinning, the effect known as twinning-induced plasticity (TWIP). The twin boundaries being strong obstacles to the motion of dislocations, their generation during plastic flow induces a dynamic Hall-Petch effect and leads to simultaneously high mechanical resistance and deformability of TWIP steels.

Another important property of these steels which, in contrast, makes difficulties for practical forming applications, is the occurrence of stress fluctuations linked to the formation and propagation of deformation bands along the deforming specimen. Mostly because of the observation of negative strain-rate sensitivity of such steels in a large range of strain rate, the plastic instability is customarily related to the Portevin-Le Chatelier (PLC) effect which stems from the interaction of dislocations with solute atoms. However, whereas this mechanism is established for high enough temperature, $T>100^{\circ} \mathrm{C}$ [2], the answer is less obvious for RT behavior. First, the bulk diffusion of carbon is too slow to account for the PLC mechanism [1]. This problem is often got round by suggesting alternative mechanisms of dynamic strain ageing of dislocations, e.g., reorientation of $\mathrm{Mn}-\mathrm{C}$ or $\mathrm{C}$-vacancy dipoles [3]. However, this suggestion does not help understanding the unusual features associated with the kine-

* corresponding author; e-mail: mikhail.lebedkin@univ-lorraine.f matics of the deformation bands and the characteristic shape of stress serrations observed at RT. Moreover, similar serrations are observed in $\mathrm{FeNiC}$ TWIP/TRIP austenitic alloys, the problem is thus more general. Particularly, the PLC effect is characterized by propagating deformation bands at high strain rate (type $A$ behavior) and a transition to static bands nucleated in a relay-race sequence (type $B$ ) or almost randomly (type $C$ ) when the applied strain rate is decreased. These changes in the spatiotemporal pattern also imply well documented changes in the shape of stress serrations [4]. In contrast, persistent propagation of deformation bands and stress serrations similar to type $A$ have been observed over more than two orders of magnitude of strain rate in the Fe22Mn0.6C steel $[5,6]$.

In the present work, an attempt is made to get an additional insight into jerky flow in the Fe22Mn0.6C steel by studying the time series characterizing the evolution of the plastic deformation at various scales of the deformation processes including macroscopically smooth and serrated flow. With this purpose in view, the local extensometry and the acoustic emission (AE) techniques are applied alongside with the conventional mechanical testing. According to the knowledge of the authors, the AE technique has not been applied up to now to study plastic deformation of FeMnC TWIP steels and the local extensometry methods were only used to characterize their serrated flow behavior [1].

\section{Experimental technique}

Flat tensile specimens of a $3 \mu \mathrm{m}$ grain size $\mathrm{Fe} 22 \mathrm{Mn} 0.6 \mathrm{C}$ steel with two gauge sections $\left(60 \times 12.6 \times 1.25 \mathrm{~mm}^{3}\right.$ and $75 \times 5.36 \times 0.6 \mathrm{~mm}^{3}$ ) were used. The specimens were tested in tension at RT with a constant crosshead velocity 
corresponding to the initial value of the imposed strain-rate $\dot{\varepsilon}_{a}$ in a range from $2.1 \times 10^{-5} \mathrm{~s}^{-1}$ to $2.5 \times 10^{-2} \mathrm{~s}^{-1}$.

The local extensometry method is described in detail in [5]. It consists in recording the displacements of a series of parallel sections of the specimen which are marked by a black-and-white contrast on its side surface. For this, one side of the specimen is painted black and a series of white stripes $1 \mathrm{~mm}$ wide and separated by $1 \mathrm{~mm}$ distance are painted over the black layer normal to the tensile (longitudinal) axis. During the tensile test, the axial positions of the markers are followed using a 1D CCD camera with a pixel size of $1.3 \mu \mathrm{m}$ and sampling frequency of $10^{3} \mathrm{~Hz}$, which is fixed relative to the immobile grip. The current drawback of this technique is the limitation of the uninterrupted recording by an interval of $600 \mathrm{~s}$, so that the complete propagation sequence of a deformation band through the series of markers could not be observed for the least value of $\dot{\varepsilon}_{a}=2.1 \times 10^{-5} \mathrm{~s}^{-1}$.

First attempts of simultaneous $\mathrm{AE}$ recording were made at $\dot{\varepsilon}_{a}=1 \times 10^{-4} \mathrm{~s}^{-1}$. The AE was measured with the aid of a Physical Acoustics LOCAN 320 system with the sampling rate of $4 \mathrm{MHz}$ and a piezoelectric transducer with a frequency band of $100-600 \mathrm{kHz}$. The transducer was clamped to the greased surface just above the deforming part of the sample. The procedure used to capture the AE events is described in this volume [7].

\section{Results and discussion}

Figure 1 illustrates the typical shape of serrations on the deformation curves and the evolution of the local strain-rate values calculated from the markers' displacements. The latter allows visualizing the kinematics of the deformation bands. It can be seen that the relatively smooth intervals on the deformation curve correspond to deformation band propagation through the entire series of local "extensometers" formed by the markers. The intervals of propagation seen in Fig. 1b are considerably shorter than the respective smooth portions in Fig. 1a. It can be suggested that each band occurs close to one specimen end and moves to the other end, passing through the extensometers. The band nucleation seemingly requires extra stresses, leading to strong stress fluctuations separating the propagation periods. Additional details of this pattern can be found in Refs. [5, 6]. Importantly, the same shape of the deformation curves, similar to the PLC instability of type $A$, is observed over the entire $\dot{\varepsilon}_{a}$-range examined. Moreover, similar local strain-rate maps are found for all strain rates, although this conclusion should be confirmed for the least $\dot{\varepsilon}_{a}$-value where the limited storage time of the CCD camera did not allow obtaining the whole propagation sequence.

Figure 2 illustrates the juxtaposition of a portion of a deformation curve with the respective AE signal. It can be recognized that the AE is particularly active during the stress fluctuations associated with the deformation band nucleation, while less $\mathrm{AE}$ is captured during the further propagation phase which is characterized by

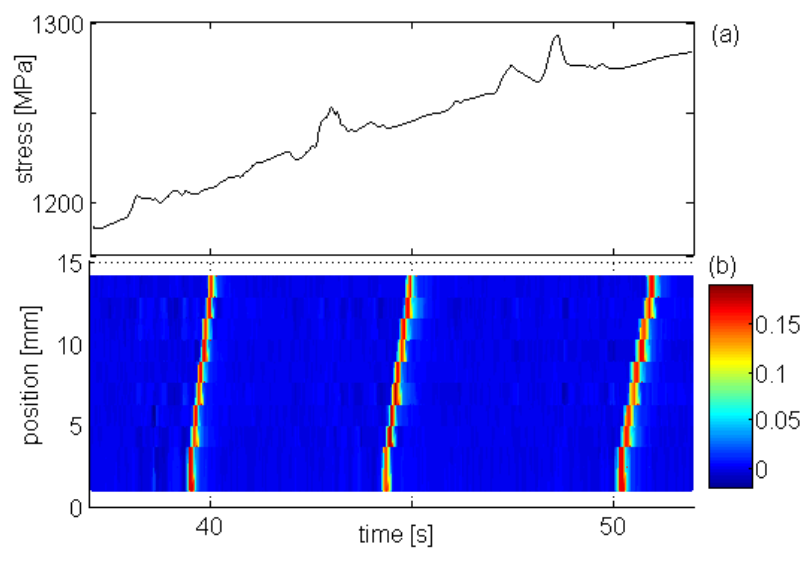

Fig. 1. (a) Part of a deformation curve confronted with (b) the local strain-rate map for $\dot{\varepsilon}_{a}=8 \times 10^{-3} \mathrm{~s}^{-1}$. The color bar displays the strain-rate scale $\left(\mathrm{s}^{-1}\right)$. It can be seen that the strain rate within the band surpasses the applied strain rate by more than an order of magnitude.

rather smooth plastic flow. However, it should be underlined that high-amplitude AE bursts occur all over the deformation curve.

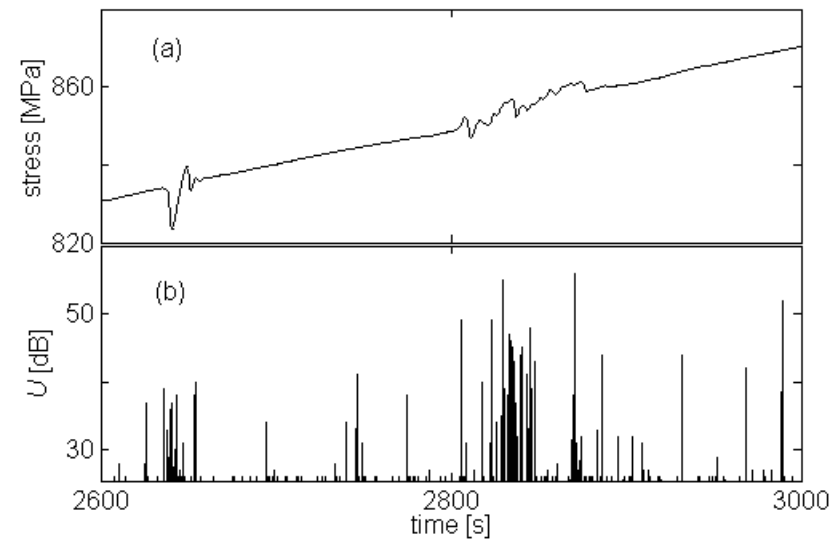

Fig. 2. (a) Part of a deformation curve and (b) the accompanying AE signal $U(t)$ for $\dot{\varepsilon}_{a}=1 \times 10^{-4} \mathrm{~s}^{-1}$.

Recent studies of the AE accompanying macroscopically smooth plastic deformation of various pure materials revealed a persistent power-law character of the statistical distributions of the AE amplitude or energy. These data bear witness to an intermittent, avalanche-like character of plastic processes at a mesoscopic scale pertaining to AE [8]. A similar value of the power-law index, $\alpha \approx 1.5$, was found for the squared amplitude of AE events in single crystals of materials with different mechanism of deformation: dislocation glide or twinning. Values in a range of 1.35 to 2 were reported in the case of polycrystals $[7,9]$. Recently, power-law behavior was also observed for all types of PLC behavior in a polycrystalline AlMg alloy [10]. However, considerably higher $\alpha$-values, typically between 2 and 3 were 
obtained in this case. It is thus of interest to apply the same analysis to the similar data for the TWIP steel. An example of such analysis is presented in Fig. 3. Surprisingly, $\alpha \approx 1.5-1.6$ is found in various strain intervals including both the almost smooth deformation following the elastoplastic transition and the stage of the developed stress serrations. A similar result was obtained in an additional test at $\dot{\varepsilon}_{a}=5 \times 10^{-5} \mathrm{~s}^{-1}$ which was performed on a different experimental installation [11]. These observations drastically differ from the results obtained for the PLC instability but are consistent with the data for the materials deformed by twinning $[7,8]$.

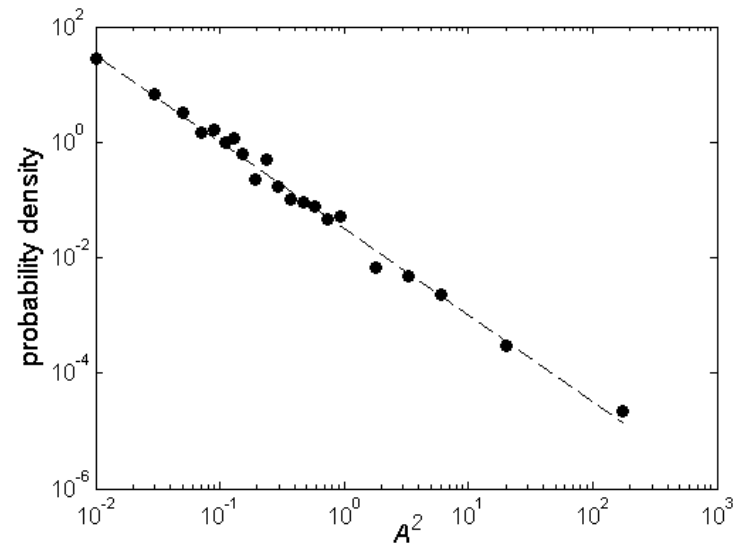

Fig. 3. Probability density function for squared amplitude of $\mathrm{AE}$ events. The estimate of the slope of the power law gives value of $1.50 \pm 0.03$.

The above description of the different aspects of the complexity of plastic deformation (stress serrations, deformation band propagation, avalanche-like AE) testifies that the plastic flow of the studied steel has a multiscale character and its apparent behavior depends on the scale of observation. Another complex phenomenon was detected in the local-extensometry tests. During the last decade, application of optical methods of local extensometry revealed a ubiquitous heterogeneity of plastic deformation which manifests itself in the form of low-amplitude and low-speed plastic strain waves [12]. The observation of such waves during deformation of various materials and in various testing conditions led to a conjecture that they are essentially governed by some generic laws independent of the specific mechanism of deformation. Plastic waves were also found in the tests presented here, although the first data obtained do not permit to either justify or falsify the last conjecture. The behavior observed is illustrated in Fig. 4 which displays an example of the strain-rate map just after elastoplastic transition and before the critical strain for the onset of the macroscopic serrations (Fig. 4b). Figure 4a shows the result of derivation of the deformation curve, $\mathrm{d} \sigma(t) / \mathrm{d} t$, which indicates the presence of the concomitant stress oscillations. The latter correspond to undulations on the $\sigma(t)$-curve, which have not attracted any attention so far, being tacitly ascribed to experimental noise. Neverthe- less, Fig. 4 shows a noticeable correlation between the strain waves and the stress oscillations. A one-to-one correspondence can hardly be expected, though, because the stress represents the global response of the sample to straining, while the strain-rate maps display the local behavior.

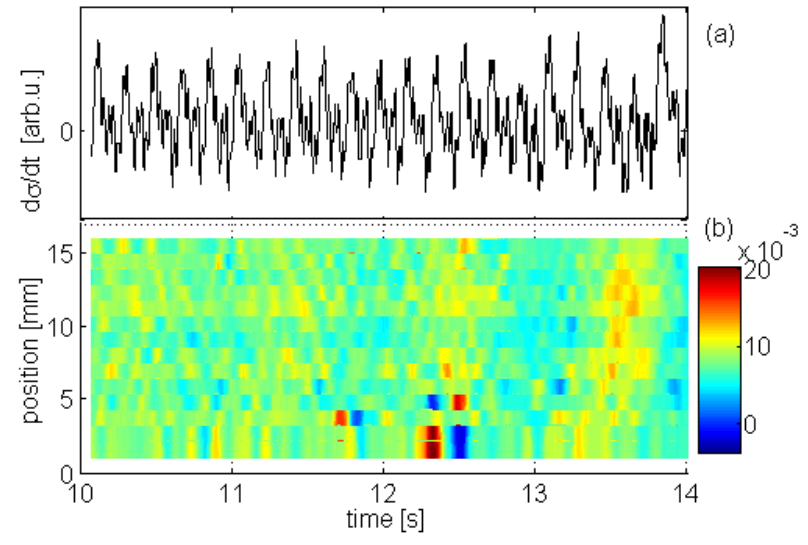

Fig. 4. (a) Blow up of the derivative of the deformation curve close to the elastoplastic transition and (b) the local strain-rate map for $\dot{\varepsilon}_{a}=8 \times 10^{-3} \mathrm{~s}^{-1}$. The map allows distinguishing several nuances: (i) localized strain-rate bursts (red to yellow spots); (ii) large clouds with a period about $1 \mathrm{~s}$, corresponding to slow modulation of the derivative oscillations; (iii) alignments of the spots indicating strain propagation through the markers's sequence and roughly corresponding to the pronounced derivative oscillations.

Clear confirmations that these oscillations reflect plastic processes were obtained using the fast Fourier transform (FFT) of $\dot{\sigma}(t)$. One particular result will be described below. For the strain rate in the range from $5.6 \times 10^{-4} \mathrm{~s}^{-1}$ to $2.5 \times 10^{-2} \mathrm{~s}^{-1}$, the FFT spectrum reveals several dominant harmonics. When one $\dot{\varepsilon}_{a}$-value is chosen as a bench mark and the time scale of other $\dot{\sigma}(t)$-curves is re-scaled in proportion to $\dot{\varepsilon}_{a}$, the positions of the peaks coincide as demonstrated in Fig. 5a. Moreover, the re-scaled frequencies coincide for the samples of two different sizes, although the specimen dimensions affect the elastic modulus of the "machine-specimen" system. Such similitude testifies to the plastic nature of the oscillations: the frequency of the processes appearing as waves in Fig. 5a adjusts itself in order to provide the plastic strain rate corresponding to $\dot{\varepsilon}_{a}$.

Furthermore, when $\dot{\varepsilon}_{a}$ is decreased down to $2.1 \times$ $10^{-5} \mathrm{~s}^{-1}$, a large spectrum without characteristic frequencies is observed (Fig. 5b). This result does not only confirm that the waves are not due to some parasite oscillations in the experimental setup. It also allows making a conjecture on the mechanism of spatial coupling in the heterogeneously deforming material. Namely, the reduced correlation between stress fluctuations, which is reflected in the large FFT spectrum, proves that such correlation is due to the internal elastic stresses generated by the local strain incompatibilities. Indeed, the influ- 


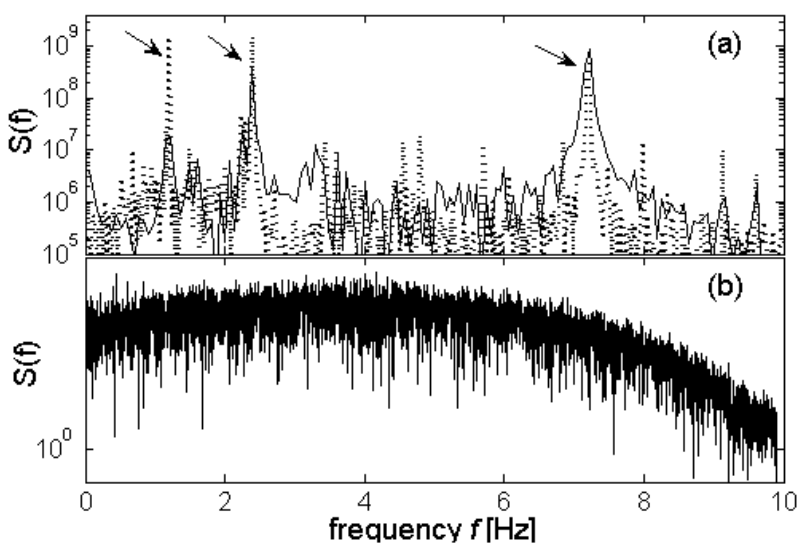

Fig. 5. (a) Parts of the FFT spectrum obtained for the derivative of the deformation curve for two applied strain-rate values: $\dot{\varepsilon}_{a}=2.2 \times 10^{-3} \mathrm{~s}^{-1}$ (solid line) and $\dot{\varepsilon}_{a}=7.8 \times 10^{-3} \mathrm{~s}^{-1}$ (dotted line). Frequency axis is rescaled for the latter curve in proportion to the ratio of the two $\dot{\varepsilon}_{a}$-values. Arrows show three superposing components. (b) Large FFT spectrum for $\dot{\varepsilon}_{a}=2.1 \times 10^{-5} \mathrm{~s}^{-1}$, which does not display characteristic frequencies.

ence of $\dot{\varepsilon}_{a}$ on the degree of correlation can be explained by a dynamical equilibrium between the heterogeneous plastic flow and the homogenization effect of plastic relaxation of the internal stresses, for which more time is available at a low strain rate.

\section{Conclusions}

The experiments carried out lead to the following conclusions:

- Several experimental facts distinguish the serrated deformation observed in the present work from the PLC effect which is often applied to explain the occurrence of plastic instability in the TWIP steels: the persistent character of the deformation band propagation which is observed over more than two orders of magnitude of strain rate, the persistent shape of the deformation curves which is consistent with the band propagation mode, and the statistical distribution of the accompanying acoustic emission which is characterized by a critical exponent similar to that for twinning or dislocation glide in pure materials but strikingly lower than the value found for an $\mathrm{AlMg}$ alloy displaying the PLC effect. Since twinning plays a substantial role in the work hardening, it is natural to suggest that twins may also be responsible for some other peculiar properties of the TWIP steels. Particularly, it would be of interest to take into account that being efficient stress concentrators, twins may emit full as well as partial dislocations into neighbouring sites and promote the deformation band propagation.

- Using the local extensometry technique, complex strain patterns including waves and localized strain-rate bursts are observed before the onset of macroscopic stress serrations. The FFT analysis of the low-amplitude undulations detected on the macroscopically smooth parts of the deformation curves and correlated with the strain waves provides a direct experimental proof, independent of models, that the spatial coupling between local strains is governed by the internal elastic stresses. This conjecture is essential for suggesting a model capable of reproducing the persistent plasticity mode observed in the TWIP steel, namely, the deformation band propagation which implies a high degree of correlation. This result may also be of interest from a more general viewpoint concerning the mechanisms of strain correlation and the observation of similar behaviors of plastic deformation of materials deforming via distinct microscopic mechanisms.

\section{Acknowledgments}

This work was supported by the program of scientific exchanges between CNRS and the Russian Academy of Sciences (project 23992) and by the French National Research Agency (ANR) under contract "PHIRCILE" (ANR 2010 JCJC 0914 01).

\section{References}

[1] O. Bouaziz, S. Allain, C.P. Scott, P. Cugy, D. Barbier, Curr. Opin. Solid State Mater. Sci. 15, 141 (2011).

[2] J. Belotteau, C. Berdin, S. Forest, A. Parrot, C. Prioul, Mater. Sci. Eng. A 526, 156 (2009).

[3] J.-K. Kim, L. Chen, H.-S. Kim, S.-K. Kim, Y. Estrin, B.C. de Cooman, Metall. Mater. Trans. A 40, 3147 (2009).

[4] P. Rodriguez, S. Venkadesan, Solid State Phenom. 42-43, 257 (1995).

[5] T.A. Lebedkina, M.A. Lebyodkin, J-Ph. Chateau, A. Jacques, S. Allain, Mater. Sci. Eng. A 519, 147 (2009).

[6] J.P. Chateau, T.A. Lebedkina, M.A. Lebyodkin, A. Jacques, S. Allain, J. Phys., Conf. Series 240, 012020 (2010).

[7] I.V. Shashkov, T.A. Lebedkina, M.A. Lebyodkin, P. Dobron, F. Chmelik, R. Kral, K. Parfenenko, K. Mathis, Acta Phys. Pol. A 122, 430 (2012).

[8] T. Richeton, P. Dobron, F. Chmelik, J. Weiss, F. Louchet, Mater. Sci. Eng. A 424, 190 (2006).

[9] T. Richeton, J. Weiss, F. Louchet, Nature Mater. 4, 465 (2005).

[10] Y. Bougherira, D. Entemeyer, C. Fressengeas, N.P. Kobelev, T.A. Lebedkina, M.A. Lebyodkin, J. Phys., Conf. Series 240, 012009 (2010).

[11] S. Allain, Ph.D. Thesis, Institut National Polytechnique de Lorraine, 2004.

[12] L.B. Zuev, Ann. Phys. 16, 286 (2007). 